\title{
EL REGISTRO DE MAMMUTHUS (PROBOSCIDEA, ELEPHANTIDAE) EN LA REPÚBLICA DE EL SALVADOR, AMÉRICA CENTRAL
}

\author{
César Laurito Mora*1\&2 \& Daniel Huziel Aguilar Calles ${ }^{3}$ \\ ${ }^{1}$ INA, Instituto Nacional de Aprendizaje - Núcleo de Turismo \\ ${ }^{2}$ Investigador Asociado-Departamento de Historia Natural, \\ Museo Nacional de CostaRica \\ Apdo. Postal 203-2200, Coronado, San José, Costa Rica \\ ${ }^{3}$ Museo de Historia Natural de El Salvador, MUHNES \\ Calle los Viveros, Colonia Nicaragua, San Salvador, El Salvador \\ *Autor para contacto: cesarlaurito@ice.co.cr
}

(Recibido: 25/05/05; aceptado: 23/02/07)

\begin{abstract}
Two new records of Mammuthus columbi Falconer (1857) are described for the Late Pleistocene of El Salvador. The Quaternary paleogeographical distribution of the Mammuthus genus in Central America is analyzed at the light of these records.

Key words: Mammals, Proboscidea, Elephantidae, Mammuthus, El Salvador, Rancholabrean.

RESUMEN: Se describen dos nuevos registros de Mammuthus columbi Falconer (1857) para el Pleistoceno Tardío de El Salvador y se analiza el patrón paleobiogeográfico de Mammuthus en el Cuaternario de América Central.

Palabras clave: Mamíferos, Proboscidea, Elephantidae, Mammuthus, El Salvador, Rancholabreano.
\end{abstract}




\section{INTRODUCCIÓN}

La fauna de proboscidios de América Central ha sido reconocida desde el siglo XIX, cuando Leidy (1859) describió un molar aislado de mastodonte procedente de la localidad de Tambla en Honduras. Durante el siglo XX los hallazgos de gomphotéridos Rhynchotheriini y Cuvieroniini hicieron de la Familia Gomphoteriidae Hay, 1922; el grupo de proboscidios de mayor distribución en América Central, con dos géneros bien reconocidos y una especie endémica Rhynchotherium blicki, Frick (1933).

América Central, también cuenta con el registro más austral en el continente Americano de la Familia Mammutidae Hay, 1922, representada por la especie Mammut americanum Kerr (Lucas \& Alvarado, 1991) que aunque muy abundante en el registro fósil de los Estados Unidos, es escaso en la República Mexicana (Polaco et al., 2001), por lo que el registro de San Pedro Sula por sí mismo es un evento extraordinario.

La Familia Elephantidae Gray, 1821, con un pobre registro fósil en América Central, fue inicialmente reconocida por Stirton \& Gealey en 1949. El material analizado procedía de la localidad Hacienda San Lorenzo, Jurisdicción de Nueva Granada, Departamento de Usulután. El material óseo y los molares asociados, se recuperaron de un banco sedimentario en las orillas del río Jerusalén y se encuentran depositados en el Museo Nacional de El Salvador. De acuerdo con dichos autores el material dental recuperado era afín a la especie Mammuthus jefersonii. Este hallazgo hizo que El Salvador fuera considerado como el límite paleobiogeográfico más austral de los Elephantidae del Nuevo Mundo.

El objetivo del presente trabajo es dar a conocer dos nuevos registros del género Mammuthus para América Central, procedentes de dos localidades diferentes de la República de El Salvador.

\section{PALEONTOLOGÍA}

El material de estudio corresponde a tres piezas dentales depositadas en el Museo de Historia
Natural de El Salvador bajo los códigos P268a, P268.2 y 2-ss-ap-30-893.

El molar P268a y el fragmento de incisivo P268.2 proceden de una localidad ubicada al pie del cerro Pacho, Cantón de Laguna Seca, Jurisdicción de Nueva Concepción, Departamento de Chalatenango (figura 1). Este fue colectado por el señor Concepción Avendaño en 1969 (com. oral Manuel Avendaño, agosto de 2000). Estos especímenes estuvieron depositados en el Museo Nacional de Antropología Dr. David J. Guzmán desde 1969 y fueron traspasados al Museo de Historia Natural de El Salvador en el año 2003.

El espécimen 2-ss-ap-30-893 corresponde a un molar fragmentado colectado por el señor Francisco Manzabares el 22 de agosto de 2002 en las márgenes del río Tomayate, a 2,30 m sobre el nivel superior del los estratos fosilíferos, cuya fauna fue descrita por Cisneros (2005), en el Municipio de Apopa, $12 \mathrm{~km}$ al norte de la ciudad de San Salvador.

\section{SISTEMÁTICA}

Se sigue la sistemática propuesta por McKenna \& Bell (1997)

Gran orden UNGULATA Ungulata Linnaeus, 1766

Mirorden ALTUNGULATA McKenna \& Bell, 1997

Orden URANOTHERIA McKenna \& Bell, 1997

Infraorden BEHEMOTA McKenna \& Bell, 1997

Parvorden PROBOSCIDEA Illiger, 1811, nuevo rango McKenna \& Bell, 1997

Superfamilia ELEPHANTOIDEA Gray, 1821

Familia ELEPHANTIDAE Gray, 1821

Subfamilia ELEPHANTINAE Gray, 1821

Tribu ELEPHANTINI Gray, 1821

Subtribu ELEPHANTIA Gray, 1821

Género MAMMUTHUS Brookes, 1828 


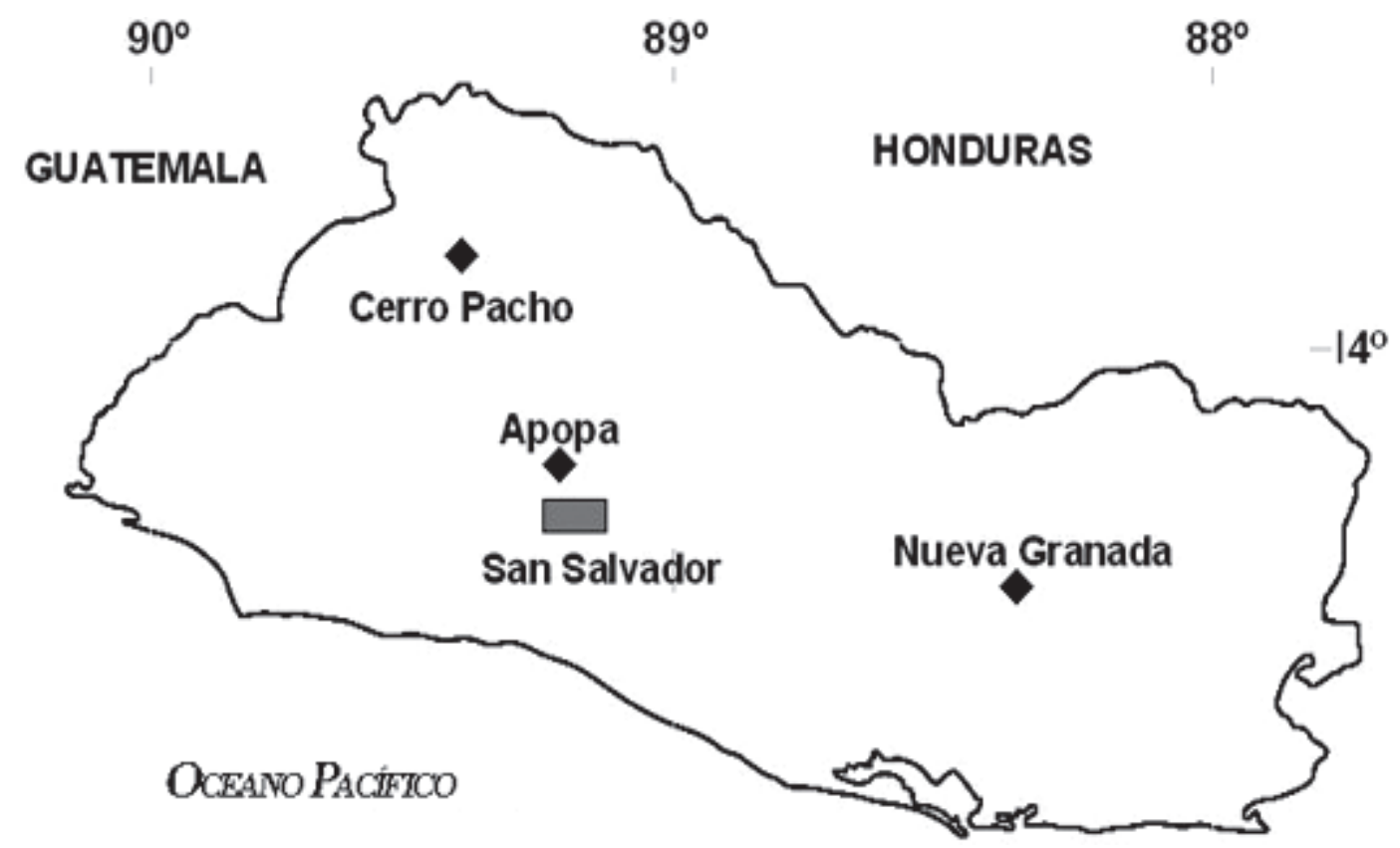

Fig. 1: Mapa de El Salvador mostrando la ubicación de las localidades donde se han hallado restos de Mammuthus columbi Falconer (1857)

Especie Mammuthus columbi Falconer (1857)

\section{$\underline{\text { Sinonimia }}$}

Muchos autores consideran que la mayoría de los registros del género Mammuthus en el Pleistoceno medio y superior de América del Norte corresponden a la especie $M$. columbi (Falconer) y asumen a $M$. jefersoni como sinónimo de $M$. columbi (Maglio, 1973 y Agenbroad, 1984).

\section{Descripción}

El espécimen P268a, corresponde a un molar inferior izquierdo con avanzado estado de desgaste. La frecuencia lamelar es de 7 lamelas cada 100 $\mathrm{mm}$; la longitud mesio-comisural de la corona varía de $10,9 \mathrm{~cm}$ en la cara vestibular a $12,8 \mathrm{~cm}$ en la cara lingual; su altura varía de $14,2 \mathrm{~cm}$ en la cara comisural a 9,01 $\mathrm{cm}$ en la cara mesial. El ancho varía de 7,6 cm en la porción distal a 9,5 $\mathrm{cm}$ en la porción media y a un ancho de $8,2 \mathrm{~cm}$ en el extremo proximal.

El espécimen P268.2, corresponde a un fragmento de incisivo de $32,2 \mathrm{~cm}$ de longitud; con diámetro proximal de $15,6 \mathrm{~cm}$ y diámetro distal de $11,43 \mathrm{~cm}$.

El espécimen 2-ss-ap-30-893, corresponde a un fragmento proximal de un molar probablemente inferior de posición incierta; frecuencia lamelar estimada de 7 lamelas cada $10.0 \mathrm{~cm}$; altura 20,01 $\mathrm{cm}$ y un ancho proximal de $6,9 \mathrm{~cm}$

\section{Discusión}

El P268a corresponde a un molar inferior por cuanto las placas lamelares son apicalmente convergentes y además, se disponen de manera casi perpendicular con respecto a la superficie oclusal, 


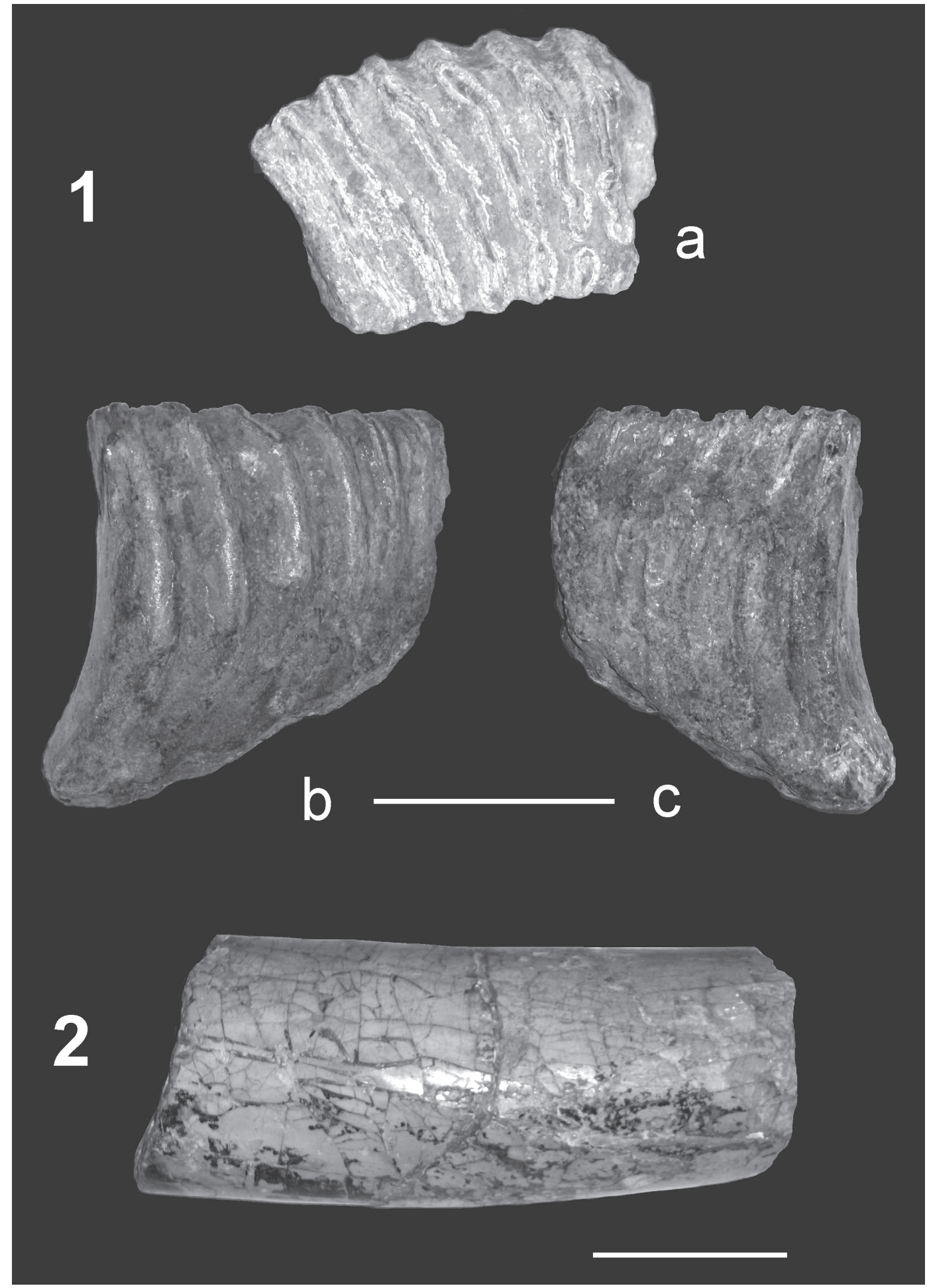

Fig. 2: 1. Molar inferior izquierdo, número de catálogo P268a en vista a: oclusal, b: lingual y c: labial, y 2. P268.2 fragmento de incisivo. Escala visual equivalente a $100 \mathrm{~mm}$. 


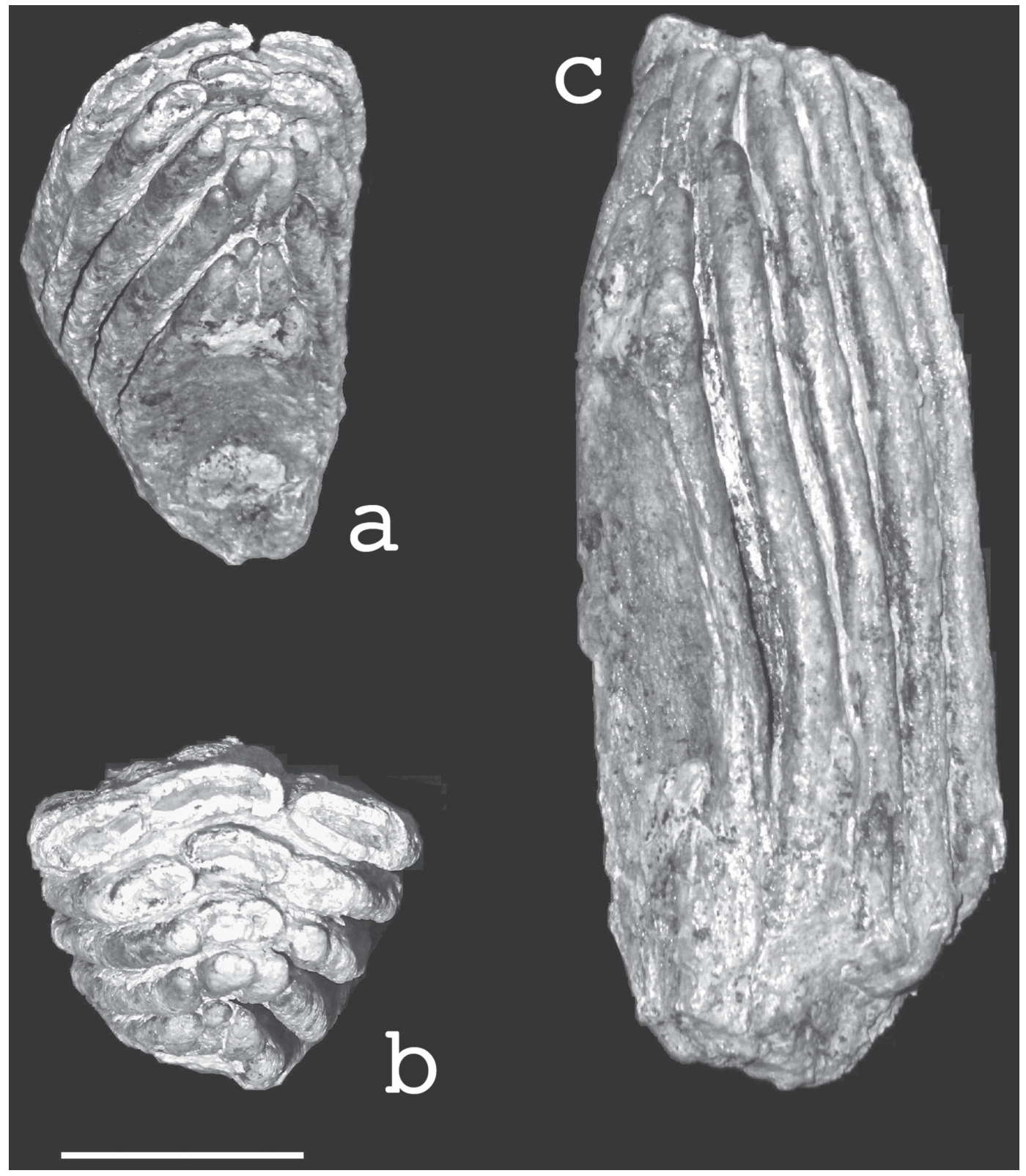

Fig. 3: fragmento proximal de un molar probablemente inferior, número de catálogo 2-ss-ap-30-893 en vista a: proximal, b. oclusal y c.labial. Escala visual equivalente a $50 \mathrm{~mm}$

criterio establecido por Maglio (1973) para distinguir los molares superiores de los inferiores en los Elephantidae.

El P268a y el 2-ss-ap-30-893 presentan una frecuencia lamelar estimada de $7 / 10 \mathrm{~cm}$, lo que nos aproxima a las especies $M$. columbi y a $M$. primigenius de acuerdo con la clave establecida por Maglio (1973). Pero Agenbroad (1984) estableció que aunque Mammuthus primigenius tuvo una amplia distribución en el territorio canadiense, Alaska, 
la región de los Grandes Lagos y el Norte de las Planicies Norteamericanas durante el Pleistoceno Tardío; esta especie nunca alcanzó el sur de Estados Unidos. Además, el ancho del molar P268a cae dentro del rango biométrico señalado por Arroyo-Cabrales et al., 2003b para la especie Mammuthus columbi.

Por otra parte, en México se han registrado solamente dos especies de Mammuthus que habitaron su territorio, a saber: Mammuthus hayi del Pleistoceno Medio con un muy escaso registro fósil y la forma Rancholabreana Mammuthus columbi (Arroyo-Cabrales et al., 2003b).

La especie $M$. hayi se distribuyó en el norte del territorio mexicano (Webb \& Dudley, 1995 y Arroyo-Cabrales et al., 2003a), en tanto que la especie $M$. columbi se distribuyó de manera más o menos uniforme a lo largo de toda la República Mexicana y tan al sur como el Estado de Chiapas (Arroyo-Cabrales et al., 2003a).

Lo anterior nos permite discriminar que los restos dentales encontrados en El Salvador corresponden a la especie Mammuthus columbi Falconer (1857).

\section{Distribución paleobiogeográfica de la especie Mammuthus columbi Falconer (1857) en América Central:}

Los especimenes descritos están constituidos principalmente por piezas dentales (ver figura 4).

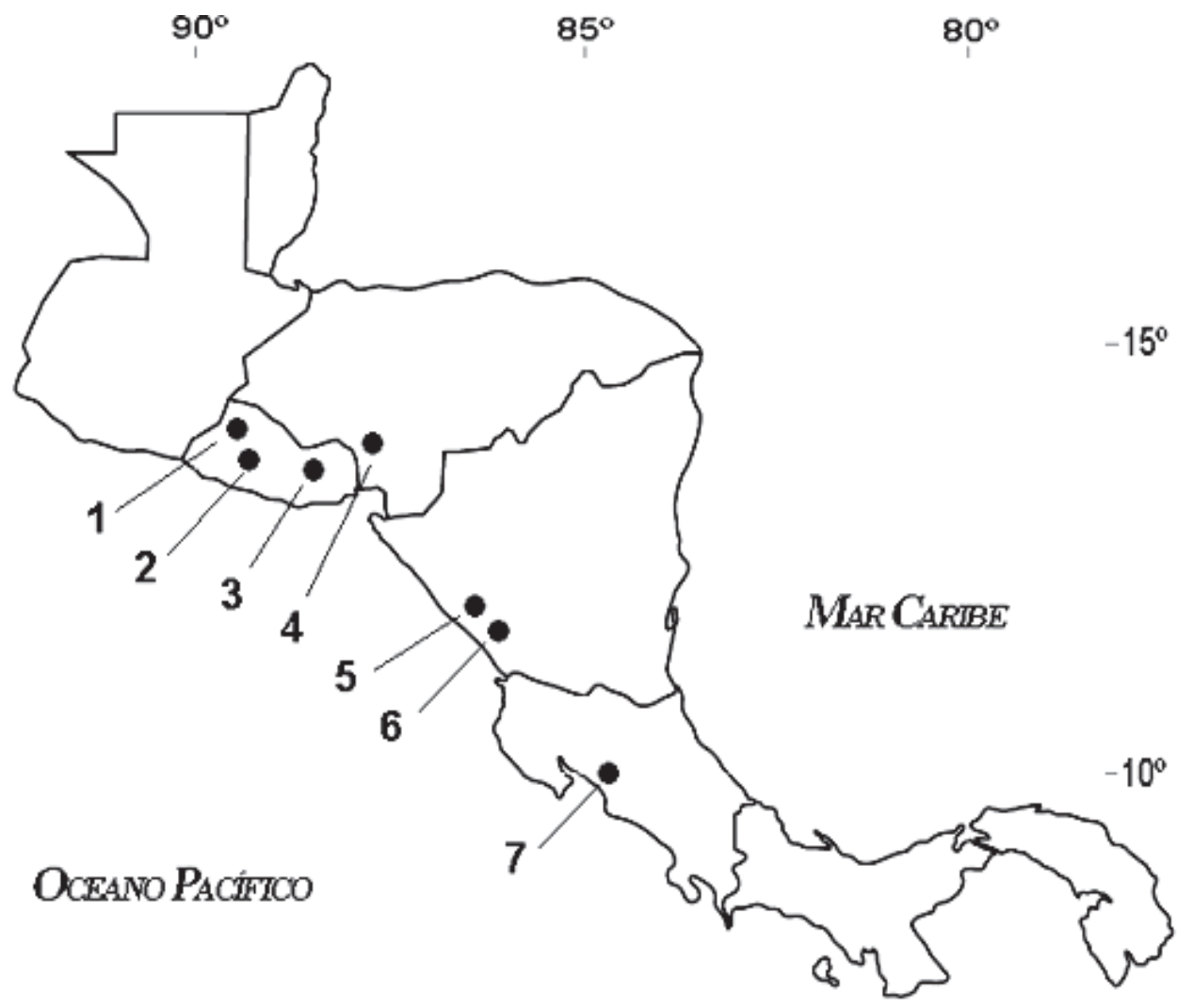

Fig. 4: Localidades de América Central donde se han hallado restos de Mammuthus: 1-3 El Salvador; 4 Honduras; 5-6 Nicaragua y 7 Costa Rica. 
Guatemala: No existen registros del género Mammuthus publicados, aunque se sabe que se cuentan con algunos registros inéditos.

El Salvador:1. Cerro Pacho, Cantón de Laguna Seca, Jurisdicción de Nueva Concepción, Departamento de Chalatenango

2. Río Tomayate, Municipio de Apopa, Departamento de San Salvador.

3. Hacienda San Lorenzo, Jurisdicción de Nueva Granada, Departamento de Usulután (Stirton \& Gealey, 1949).

Honduras: 4. Orillas del río Humuya, Municipio de Humuya, Departamento Comayagua (Webb \& Perrigo, 1984).

Nicaragua: 5. Estrecho de Rivas, en las cercanías de Masachapa, en la costa Suroeste de Nicaragua (Espinosa, 1976), nunca fue descrito en detalle, ahora está extraviado.

6. Estrecho de Rivas, Sitio Paleontológico Los Mora, rivera del río San Luis, afluente del río Tola en la localidad del mismo nombre (García, 1996).

Costa Rica: 7. Localidad de Orotina, provincia de Alajuela, un molar recuperado en 1963 (Laurito, 1988 y Lucas et al., 1997) el ejemplar fue enviado a Los Angeles County Museum y en la actualidad está extraviado.

Los hallazgos parecen concentrarse en las zonas bajas occidentales del istmo centroamericano. Tal sesgo en la distribución, podría deberse a que el desarrollo urbano e industrial se localiza a lo largo del pacífico centroamericano, especialmente entre Nicaragua y Guatemala.

Otra posibilidad es que durante el Pleistoceno Superior, las sabanas arboladas asociadas al bosque seco tropical, se restringieran al pacífico de América Central, lo que permitió a estos elefantes migrar a lo largo de este corredor biológico. En la actualidad los relictos de bosque seco tropical en América Central se localizan a lo largo de la costa pacífica, entre el Norte de Costa Rica, Suroeste de Nicaragua y la costa pacífica de El Salvador. Ello explicaría el porqué no se han encontrado restos de Mammuthus en el Caribe de América Central y los estados mexicanos de Yucatán o Quintana Roo.

\section{$\underline{\text { Edad }}$}

La distribución estratigráfica de la especie Mammuthus columbi Falconer (1857) en América del Norte se acepta como Pleistoceno Tardío o Rancholabreano (Kurtén \& Anderson, 1980 y Lundelius et al., 1987). En el territorio mexicano, esta edad se asume más por asociación faunal que por dataciones radiométricas.

En el caso de los ejemplares P268a y P268.2 no existe control estratigráfico, pero se asume una edad NALMA Rancholabreano.

En cuanto al ejemplar 2-ss-ap-30-893 recuperado en la rivera del río Tomayate, se cuenta con un control estratigráfico local y queda claro que procede de los depósitos volcaniclásticos de la Formación Cuscatlán y no de las secuencias sedimentarias infrayacentes, portadoras de una importante fauna de vertebrados descritas por Cisneros, 2005, quien les asigna una edad Pleistoceno Temprano a Medio. Debido a lo anterior se asume una edad NALMA Rancholabreano.

\section{CONCLUSIONES}

Se describen dos nuevos registros de Mammuthus columbi Falconer (1857) para la República de El Salvador y América Central. La edad asumida para estos hallazgos es Pleistoceno Superior o edad NALMA Rancholabreana.

Los registros del género Mammuthus se distribuyen geográficamente en la vertiente pacífica del istmo centroamericano, patrón que se replica en el sur de la República Mexicana. Este patrón paleobiogeográfico coincide con la actual distribución relictual del Bosque Tropical Seco del pacífico centroamericano.

La localidad de Orotina, en el Pacífico Central de Costa Rica representa el límite paleobiogeográfico más austral de los Elephantidae en el Nuevo Mundo y en particular de la especie Mammuthus columbi Falconer (1857). 


\section{AGRADECIMIENTOS}

Al Dr. Joaquín Arroyo Cabrales del Instituto Nacional de Antropología e Historia de México (INAH) y a la Dra. Eileen Johnson de Texas Tech University, Lubbock; quienes gentilmente suministraron parte de la bibliografía utilizada en este trabajo.

AlageólogaAnaLucía ValeriodelDepartamento de Historia Natural del Museo Nacional de Costa Rica, por el apoyo logístico brindado durante la realización de esta investigación.

\section{REFERENCIAS}

AGENBROAD, L.D., 1984: New World mammoth distribution. En Martin, P.S. \& Kein, R.G. (Eds.): Quaternary Extinctions: A Prehistoric Revolution. Univ. Arizona Press. Tucson.

ARROYO-CABRALES, J., POLACO, O. J., JOHNSON, E. \& GUZMÁN, A. F., 2003a: The distribution of the genus Mammuthus in México. En J. W. F. Reumer, J. De Vos \& D. Mol, (Eds.): Advances in Mammoth Research (Proceedings of the Second International Mammoth Conference, Rotterdam, May 16-20 1999). DEINSEA, 9: 27-39.

ARROYO-CABRALES, J., POLACO, O. J. \& AGUILAR-ARELLANO, F. J., 2003b: Remains of Mammuthus housed in the collections of the Instituto Nacional de Antropología e Historia, México. En J. W. F. Reumer, J. De Vos \& D. Mol, (Eds.): Advances in Mammoth Research (Proceedings of the Second International Mammoth Conference, Rotterdam, May 16-20 1999). DEINSEA, 9: 17-25.

CISNEROS, J.C., 2005: New Pleistocene Vertebrate Fauna from El Salvador- Re. Bras. de Paleontol., 8(3): 239-255.

ESPINOSA ESTRADA, J. 1976: Excavaciones arqueológicas en "El Bosque". Instituto
Geográfico Nacional, Managua, 76 pp. (Informe 1).

GARCÍA, R., 1996: Sitio Paleontológico Los Mora. Instituto Nicaragüense de Cultura. 5 pp. (informe inédito).

KURTÉN,B.\&ANDERSON,E., 1980: Pleistocene Mammals of North America. Columbia University Press. New York, 422 pp.

LAURITO, C.A., 1988: Los Proboscidios fósiles de Costa Rica y su contexto en la América Central-Vínculos: Revista de Antropología del Museo Nacional de Costa Rica 14:29-58.

LUCAS, S.G. \& ALVARADO, G.E., 1991: El hallazgo más austral de Mammut americanum: el caso del mastodonte de San Pedro Sula, Honduras- Rev. Geol. Amér. Central, 13: 85-89.

LUCAS, S.G.; ALVARADO, G.E. \& VEGA, E. 1997. The Pleistocene mammals of Costa Rica- Journal of Vertebrate Paleontology, 17 (2):413-427.

LUNDELIUS, E.L., DOWNS, T., LINDSAY, E.H., SEMKEN, H.A., ZAKRZEWSKI, R.J., CHURCHER, C.S., HARRINGTON, C.R., SCHULTZ, G.E. \& WEBB, S.D., 1987: The North American Quaternary Sequence. En Woodburne, M.O. (Ed.): Cenozoic Mammals of North America. Univ. California Press. Berkeley.

MAGLIO, V.J., 1973: Origin and evolution of the Elephantidae.- Trans. Amer. Philos. Soc., New Ser. 63(3):1-149.

MCKENNA, M. \& BELL, K., 1997: Classification of Mammals above the specie level. Columbia University Press, New York, $631 \mathrm{pp}$.

STIRTON, R.A. \& GEALEY, W.K., 1949: Reconnaissance geology and vertebra- 
te paleontology of El Salvador, Central America- Geological Society of America Bulletin, 60:1731-1764.
WEBB, S.D. \& DUDLEY, J.P., 1995: Proboscidea from the Leisey Shell Pits, Hillsborough County, Florida. Bull. Florida Mus. Nat. Hist. 37(20):645-660. 
